\title{
2D Falicov-Kimball Model in the Perturbative Regime at Finite Temperatures
}

\author{
L. DȨBSKI* \\ Institute of Physics, Adam Mickiewicz University \\ Umultowska 85, 61-614 Poznań, Poland
}

\begin{abstract}
Finite-temperature properties of the Falicov-Kimball model in two approximations were studied in the perturbative regime, i.e. for $t / U \ll 1$, where $t=1$ is the hopping constant and $U=10$ denotes the Coulomb interaction strength. In our study, we determined the phase diagram of the model in the second order of the perturbation theory, where it reduces to the antiferromagnetic Ising model in the emergent magnetic field. In the fourth order, where our model constitutes the Ising model with more complicated frustrated antiferromagnetic interactions, the phase diagram was established. The Monte Carlo method was employed to investigate the phase transition lines. The existence of stripe ordering at finite temperatures is proved.
\end{abstract}

PACS numbers: 71.10.Hf, 75.10.Hk, 75.30.Kz, 75.40.Mg

\section{Introduction}

The Falicov-Kimball model (FKM) [1] is one of simplest possible lattice model of itinerant interacting fermions. In this model, two sorts of particles are present: "heavy" particles described by occupation number $w_{x}$, which can take two values: 0 and 1 , and quantum itinerant spinless fermions, described by creation and annihilation operators $c_{x}^{\dagger}, c_{x}$. The Hamiltonian, defined on the subset $\Lambda$ of the lattice, is

$$
\begin{aligned}
& H_{\Lambda}\left(\left\{w_{x}\right\}\right)=-\sum_{x, y \in \Lambda} t_{x y} c_{x}^{\dagger} c_{y}+U \sum_{x \in \Lambda} w_{x} n_{x} \\
& +\sum_{x \in \Lambda} \mu_{+} w_{x}+\sum_{x \in \Lambda} \mu_{x} c_{x}^{\dagger} c_{x} .
\end{aligned}
$$

In the formula above, $t_{x y}$ are hopping constants (in our case much smaller than the strength of the Coulomb interaction $U$ ) and we assume here that they are equal to $t$, if $x, y$ are nearest-neighbors (nn) and zero otherwise. $\mu_{+}, \mu_{-}$are chemical potentials for heavy and itinerant particles, respectively.

This model has been used to describe numerous phenomena in solid state physics: semiconductor-metal transitions, appearance of ordering(s) in mixed-valence compounds, non-resonant Raman scattering [2].

Moreover, the behavior of the FKM can serve as a guide in investigation of the Hubbard model, where exact results still are rare. For instance, the segregation observed in FKM would correspond to ferromagnetism in the Hubbard model [3].

There exist numerous both rigorous and numerical results on the area of the FK model. Most of them concern orderings in ground states and in low temperatures (for representative sample of rigorous results see [4]). How-

\footnotetext{
* e-mail: lech.debski@interia.eu
}

ever, temperature-driven phase transitions are much less known.

This opportunity motivated us to investigate this problem. We begin the study from the simplest non-trivial case, i.e. two-dimensional model at half-filling in the perturbative regime. In this range of parameters one can use perturbation expansion. In the second order of perturbation theory, one obtains the antiferromagnetic nn Ising model with the Hamiltonian

$$
H_{\mathrm{eff}}^{(2)}=h \sum_{i} s_{i}+2 \frac{t^{2}}{U} \sum_{d(i, j)=1} s_{i} s_{j}
$$

$\left(s_{i}\right.$ in the formula above is related to $w_{i}$ by $\left.s_{i}=w_{i}-1 / 2\right)$. In the fourth order one obtains the Ising model with complicated frustrated short range (up to two lattice spacings) interactions

$$
\begin{gathered}
H_{\mathrm{eff}}^{(4)}=h \sum_{i} s_{i}+\left(2 \frac{t^{2}}{U}-18 \frac{t^{4}}{U^{3}}\right) \sum_{d(i, j)=1} s_{i} s_{j} \\
+\frac{6 t^{4}}{U^{3}} \sum_{d(i, j)=\sqrt{2}} s_{i} s_{j}+\frac{4 t^{4}}{U^{3}} \sum_{d(i, j)=2} s_{i} s_{j} \\
+\frac{40 t^{4}}{U^{3}} \sum_{\mathcal{P}_{4, i j k l}} s_{i} s_{j} s_{k} s_{l}+\frac{3 t^{4}}{2 U^{3}} \sum_{\mathcal{P}_{4, i j k l}} \mathbf{1}
\end{gathered}
$$

$\left(\mathcal{P}_{4, i j k l}\right.$ is a unit square plaquette spanned by sites $i, j, k, l)$. We study this effective model with the use of Monte Carlo method - perhaps the most universal method in the area of spin systems. Ground state orderings [5] presented in Fig. 1 are our reference point.

\section{The Monte Carlo simulations}

The Monte Carlo (MC) simulations presented here are adapted from the simulations presented in [6]. We have generated equilibrium configurations of the finite- 
-size square spin samples of the size $L \times L(L \leq 54)$ for fixed values of the model parameters. algorithm. Periodic boundary conditions were imposed and thermalization of the initial configurations of the length of $10^{5}$ to $10^{6}$ Monte Carlo steps (MCS) was applied, depending on the size of a sample. The 48-bit random number generator was used. Each MC run was split into $k(6 \leq k \leq 40)$ segments consisting of $10^{7}$ MCS to calculate partial averages. In the calculation of the partial averages only every $i$-th MC step contributes $(6 \leq i \leq 10)$, to avoid correlations between sampled configurations of spins in the system.

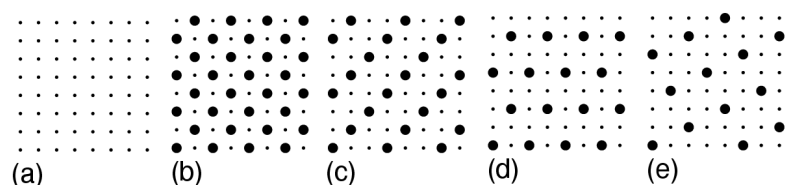

Fig. 1. The ground state orderings for effective Hamiltonian (3) in the fourth order of the perturbation theory. The configurations of the heavy particles (marked by bold dots •) in parts (a), (b), (c), (d) and (e) correspond to the phases referred as (0), (1), (2), (3) and (4), respectively.

To find the phase transition points, the Binder cumulant [6] $Q_{L}=\left\langle M_{\alpha}^{2}\right\rangle_{L}^{2} /\left\langle M_{\alpha}^{4}\right\rangle_{L}$ was used. Here $\left\langle M_{\alpha}^{n}\right\rangle_{L}$ denotes the $n$-th power of the $\alpha$ spins order parameter, averaged over an assembly of independent samples of the size $L \times L$. We call this analysis the intersection method.

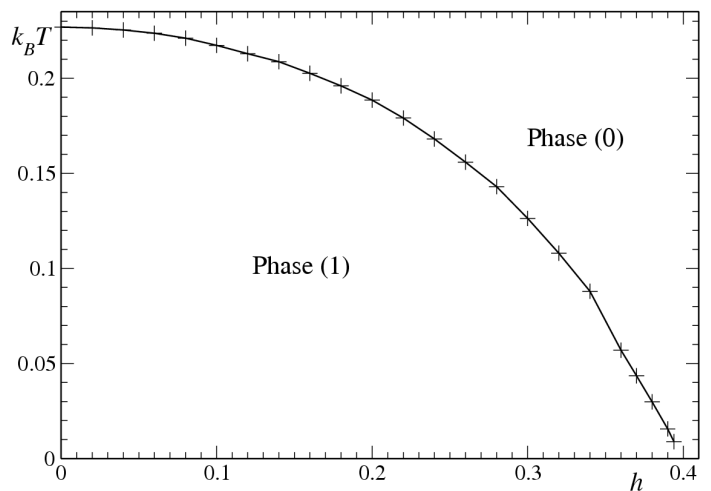

Fig. 2. The phase diagram of the 2D Falicov-Kimball model on a square lattice in the second order of perturbation theory. $h$ denotes the difference of the chemical potentials $\mu_{+}$and $\mu_{-}$for heavy and itinerant particles, respectively. The points, for which the calculations were performed within this paper, are marked with $\times$ 's and the lines are drawn to guide eyes. Labels of phases are explained in the caption of Fig. 1.

Assuming $t / U=1 / 10$ and applying this method, we obtained the phase diagram of the model with Hamiltonian (2) which is presented in Fig. 2. Together with the ground state orderings [5] (Fig. 1), it makes the reference

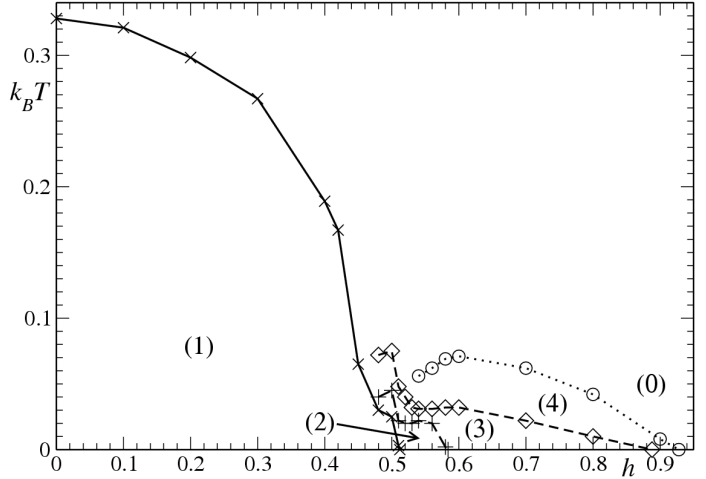

Fig. 3. The sketch of the phase diagram of the 2D Falicov-Kimball model on a square lattice in the fourth order of perturbation theory. The points, for which the calculations were performed within this paper, are marked with symbols and the lines are drawn to guide eyes. Labels of phases are explained in the caption of Fig. 1.

point for investigation of the model with Hamiltonian (3). In our study of the latter model, we started from the calculation of the phase transition point at $h=0$. Using the intersection method, we obtained the critical value $k_{\mathrm{B}} T=0.3280(2)$, marked with $\times$ in Fig. 3 .

To determine the way of ordering in the system, we investigated the behavior of the cumulant $Q$ with the use of each of the two alternative sublattices (see Fig. 1b), which implied the presence of phase (1). This fact was confirmed by the behavior of the cumulant $Q$ determined with the use of each of the three alternative sublattices (see Fig. 1c). We observed the parallel horizontal curves for each value of the system size $L$. Analogously we determined the critical values of $k_{\mathrm{B}} T$ for the phase transitions at $h=0.1$ to 0.45 . The behavior of the cumulant $Q$ gave no evidence of phase (2) illustrated in Fig. 1c.

Only for $0.48 \geq h \geq 0.45$ we observed small oscillations of the values of the Binder cumulants $Q$ having divided the lattice into two or into three sublattices, signalizing an increase in the contribution of the further terms in Hamiltonian (3) to the interaction energy of degrees of freedom, although in the latter case the lines for different $L$ 's still did not intersect, which meant that phase (2) was not realized.

We univocally detected phase (2) and the next ones presented in Fig. 1 only in the simulations for $h \geq 0.48$. Figures 4 and 5 show the exemplary $k_{\mathrm{B}} T$ dependence of the cumulants $Q$ at $h=0.54$. We can conclude that for $k_{\mathrm{B}} T \leq 0.022$ phase $(2)$ appears. It follows from the dependences $Q\left(k_{\mathrm{B}} T\right)$ for various $L$, with the use of each of the two alternative sublattices, as illustrated in Fig. 4. The oscillations of the values of $Q$ and the appearance of intersections of the dependences $Q\left(k_{\mathrm{B}} T\right)$ for even $L$ values, illustrated in Fig. 4 and for odd $L$ values, illustrated in Fig. 5, show that phase (3) appears whose upper boundary point can be estimated as about $k_{\mathrm{B}} T=0.031$. 


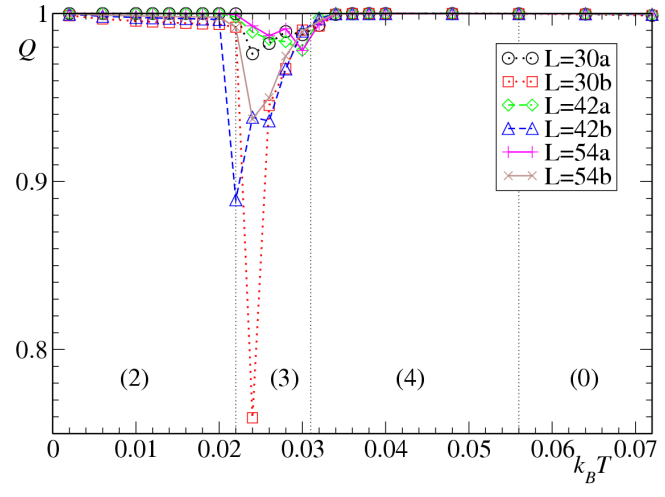

Fig. 4. The $k_{\mathrm{B}} T$ dependence of two Binder cumulants $Q$ calculated for the samples with different linear size $L$, listed in the legend box, at $h=0.54$. Each cumulant $Q$ was calculated from the one of two sublattices shown in Fig. 1b. Phase (1) is absent here.

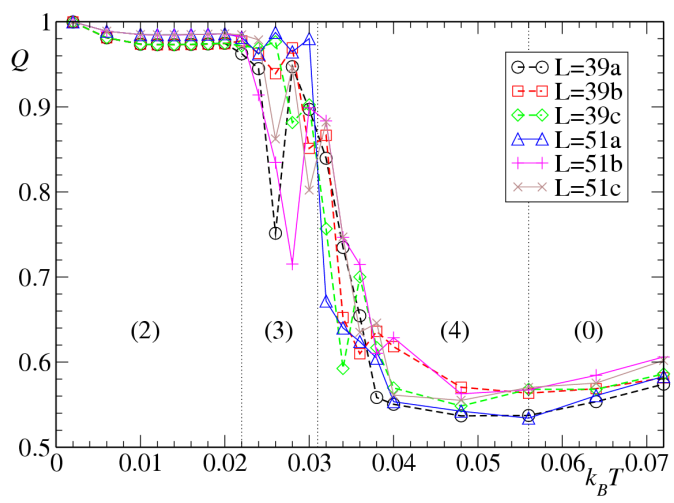

Fig. 5. The $k_{\mathrm{B}} T$ dependence of three Binder cumulants $Q$ calculated for the odd samples. Each cumulant $Q$ was calculated from the one of three sublattices shown in Fig. 1c. Phase (1) is absent here.

For higher values of $k_{\mathrm{B}} T$ between 0.031 and 0.056 phase (4) appears. Its appearance is reflected by the increase in values of $Q$ presented in Fig. 4 and by intersections of $Q$ values in Fig. 5. Above this region which the disordered phase (0) appears. It is evident that the appearance of phases (3) and (4) influences the behavior of the cumulants explained in the captions of Figs. 4 and 5 which allowed us to estimate the boundaries of these phases.
It is worth noting that for $k_{\mathrm{B}} T=0$ the ground state boundaries between phases calculated theoretically in [7] are used, respectively $h=0.512,0.584,0.888$ and 0.928 (see Fig. 3). They very well complement the boundaries obtained here by the Monte Carlo simulations. Thus we may conclude that the ground state boundaries calculated by Datta et al. [4] of $h=0.316,0.352,0.384$ and 0.404 , respectively, are hardly confirmed.

\section{Conclusions}

This paper presents our MC results which allowed us to establish the finite-temperature phase diagram of the Falicov-Kimball model on the square lattice in the perturbative regime. Although these results in part concerning the phases (3) and (4) are based on indirect conclusions, our analysis evidently proves the existence of stripe ordering also at finite temperatures in the FKM. The earlier papers showed their existence at low $T$ but did not tell anything about the value of the critical temperature(s). As far as we know, the only exception is the Néel phase [2], the simplest case of our analysis.

\section{Acknowledgments}

The authors wish to thank Professors G. Musial and J. Wojtkiewicz for valuable discussions, and the State Committee for Scientific Research for partial financial support within the grants MEIN 24/6 PR UE/2006/7.

\section{References}

[1] L.M. Falicov, J.C. Kimball, Phys. Rev. Lett. 22, 997 (1969).

[2] J. Freericks, V. Zlatić, Rev. Mod. Phys. 75, 1333 (2003).

[3] J.K. Freericks, E.H. Lieb, D. Ueltschi, Commun. Math. Phys. 227, 243 (2002).

[4] N. Datta, R. Fernandez, J. Fröhlich, J. Statist. Phys. 96, 545 (1999).

[5] R. Lemański, J. Wojtkiewicz, Phys. Status Solidi B 236, 408 (2003).

[6] G. Musial, Phys. Rev. B 69, 024407 (2004).

[7] J. Wojtkiewicz, J. Statist. Phys. 112, 1049 (2003). 